\title{
Characterization of integrons in Burkholderia cepacia clinical isolates
}

\author{
Linda Furlanis, Lucia Corich, Lucilla Dolzani, Enrico Angelo Tonin, Cristina Lagatolla \\ Dipartimento di Scienze della Vita, Università degli Studi di Trieste
}

Key words: Burkholderia cepacia complex, integrons, antibiotic resistance, Cystic Fibrosis, aacA4-lb

Caratterizzazione di integroni presenti in isolati clinici di Burkholderia cepacia

\section{SUMMARY}

Burkholderia cepacia is an opportunistic pathogen able to colonize the airways of Cystic Fibrosis (CF) patients, frequently developing chronic infections. In $20 \%$ of cases these infections cause severe and poorly controlled pathological situations because of the intrinsic antibiotic resistance expressed by the microorganism. CF patients are often subjected to antibiotic therapy: this facilitates the acquisition of antibiotic resistance determinants by the infecting bacteria. Integrons are mobile genetic elements that are widespread in bacterial populations and favor the acquisition of gene cassettes coding for these determinants. The presence of class I integrons was investigated by PCR with primers specific for the 5' and 3' ends in Burkholderia isolates recovered from patients in treatment at the CF center of Friuli Venezia Giulia.

The same integron, carrying an uncommon allelic form (Ib) of the aacA4 gene in its cassette array and conferring resistance to some aminoglycosides, was found in two independent isolates (different RAPD profiles) infecting two different patients. In both isolates the integron was carried by plasmids and was still present 3 and 6 years later the first finding. Despite the exchange of integrons between bacterial pathogens is fully described, these items were not frequently found in Burkholderia isolates. Although the clinical relevance of the integron we identified is low (a single gene cassette encoding a widespread resistance), we feel concerned that these genetic elements begin to circulate in this bacterial species, as this could make more and more troublesome the treatment of infections notoriously difficult to eradicate.

Burkholderia cepacia è un patogeno opportunista capace di colonizzare le vie aeree dei pazienti affetti da Fibrosi Cistica (FC) sviluppando frequentemente infezioni croniche. Nel 20\% dei casi queste infezioni determinano quadri clinici temibili e scarsamente controllabili a causa dell’intrinseca resistenza agli antibiotici manifestata dal microrganismo. I pazienti FC sono spesso sottoposti a terapia antibiotica: questo favorisce l'acquisizione di determinanti per l'antibiotico-resistenza da parte dei batteri che li colonizzano. Uno dei meccanismi che favorisce l'aumento di antibiotico resistenze è la presenza di integroni, elementi genetici molto diffusi nelle popolazioni batteriche, che favoriscono l'acquisizione di cassette geniche codificanti per tali determinanti. I ceppi utilizzati in questo lavoro sono stati isolati dall'espettorato di pazienti affetti da FC afferenti al Centro Regionale per la Fibrosi Cistica del Friuli Venezia Giulia dal 2000 al 2006 (Tabella 1). Acinetobacter baumannii 204A è stato utilizzato come controllo positivo avente la cassetta genica aacA4-Ib wt (3). La tipizzazione dei ceppi è stata eseguita con il metodo Random Amplified Polymorphic DNA (RAPD) secondo quanto descritto da Mahenthiralingam e collaboratori (5). La reazione di amplificazione della regione variabile dell’integrone è stata eseguita come descritto da Lévesque e collaboratori (4). Le reazioni di sequenziamento sono state effettuate presso il BMR Genomics (Padova) e le sequenze ottenute sono state appaiate con la banca dati fornita dall'NCBI. Entrambe le forme alleliche Ib e Ib' sono state clonate in pUC19 dopo amplificazione in E. coli DH5 $\alpha$. I cloni contenenti il plasmide ricombinante sono stati selezionati e mantenuti in terreno LB contenente ampicillina $100 \mu \mathrm{g} / \mathrm{mL}$. La valutazione della MIC per gli amminoglicosidi cui la cassetta aacA4 conferisce resistenza (7), è stata eseguita in solido secondo il protocollo descritto dal CSLI 2008 (2). Il Southern blot è stato eseguito con il metodo descritto da Sambrook e collaboratori (6).

La presenza di integroni è stata investigata, mediante PCR con primer omologhi alle regioni costanti (CS) delle estremità 5' e 3' (Figura II), in ceppi di Burkholderia isolati da pazienti afferenti al Centro FC del Friuli Venezia Giulia (Tabella 1). Gli isolati ottenuti da due pazienti (A e B) sono risultati colonizzati da ceppi indipendenti, non correlati dal punto di vista epidemiologico e distinguibili mediante RAPD analysis (Figura I), ma portatori dello stesso integrone (Figura II): il sequenziamento della regione variabile ha rivelato infatti la stessa cassetta genica, la forma allelica Ib' del gene aacA4, codificante per la resistenza ad alcuni aminoglicosidi (Figura III). La clonazione della cassetta in E. coli DH5 $\alpha$ ha permesso di valutare l'effetto della mutazione puntiforme ritrovata in questo gene rispetto alla forma wild tipe più frequentemente isolata (Tabella 2). In entrambi gli isolati si è dimostrato tramite Southern blot che l'integrone è portato da un plasmide ed è risultato presente in prelievi successivi, a 3 e a 6 anni di distanza (Figura IV). Inoltre è stata riscontrata una differenza nella grandezza dei plasmidi in cui l'integrone è presente, anche da isolati provenienti dallo stesso paziente; ciò dimostra una notevole plasticità del genoma di questi microrganismi, proprietà che determinerebbe un ulteriore vantaggio nell'acquisizione di nuovi frammenti genici importanti dal punto di vista patogenetico.

La circolazione degli integroni nelle popolazioni batteriche che interessano la patologia umana sia ampiamente documentata, questi elementi genici non sono stati frequentemente ritrovati in ceppi di Burkholderia. L'integrone da noi identificato non è clinicamente molto rilevante (un'unica cassetta genica codificante per una resistenza molto diffusa), ma riteniamo preoccupante il fatto che questi elementi genici inizino a circolare in questa specie batterica, per le complicazioni che questo potrebbe avere in futuro sulle possibilità terapeutiche nei confronti di infezioni già difficili da eradicare.

Tabella I. Ceppi di B. cepacia complex (Bcc) raccolti da pazienti FC nel corso degli anni 2000-2006

\begin{tabular}{llll}
\hline & $\begin{array}{l}\text { Isolati } \\
\text { anno 2000 }\end{array}$ & $\begin{array}{l}\text { Isolati } \\
\text { anno 2003 }\end{array}$ & $\begin{array}{l}\text { Isolati } \\
\text { anno 2006 }\end{array}$ \\
\hline PAZIENTE A & $\begin{array}{l}\text { BTSI } \\
\text { BTS2 }\end{array}$ & BTS25 & BTS28 \\
PAZIENTE B & $\begin{array}{l}\text { BTS1I } \\
\text { BTS20 }\end{array}$ & \\
& BTS12 & BTS21 & - \\
PAZIENTE C & BTS5 & BTS26 & - \\
& & BTS23 & \\
PAZIENTE D & BTS3 & BTS24 & - \\
PAZIENTE E & BTS4 & BTS22 & -
\end{tabular}

Tabella 2. Modificazione del profilo di resistenza di E. coli nei confronti di alcuni aminoglicosidi dopo clonazione delle cassette geniche aacA4-Ib (wt) e aacA4-Ib' (mutata).

Le MIC sono espresse in $\mu \mathrm{g} / \mathrm{mL}$.

\begin{tabular}{llll}
\hline & $\mathrm{DH} 5 \alpha$ & $\begin{array}{l}\mathrm{DH} 5 \alpha / \\
\mathrm{pUC19} \text {-Int204A (wt) }\end{array}$ & $\begin{array}{l}\mathrm{DH} 5 \alpha / \\
\mathrm{pUCI} \text { 9-Int I (mutata) }\end{array}$ \\
\hline Netilmicina & 0.5 & 8 & 2 \\
\hline Kanamicina & $\mathrm{I}$ & 32 & 32 \\
\hline Amikacina & 0.5 & 4 & $\mathrm{I}$ \\
\hline Tobramicina & 0.25 & 8 & 4 \\
\hline
\end{tabular}




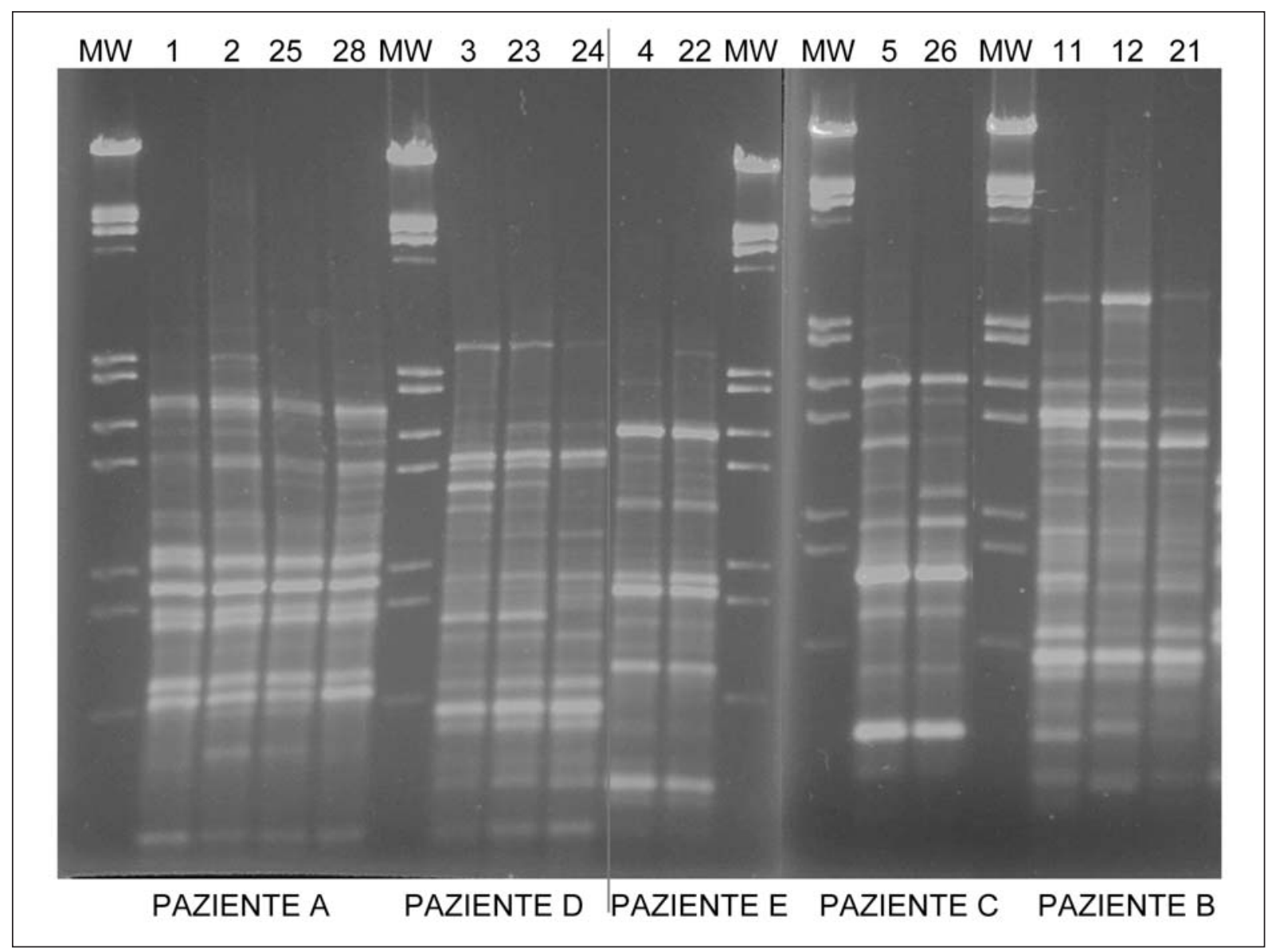

Figura I. Profilo elettroforetico degli amplificati ottenuti mediante RAPD analysis con primer 208.

$8 \mu \mathrm{L}$ della miscela di reazione sono stati separati in gel di agarosio al 2\%; MW: DNA Molecular Weight Marker III

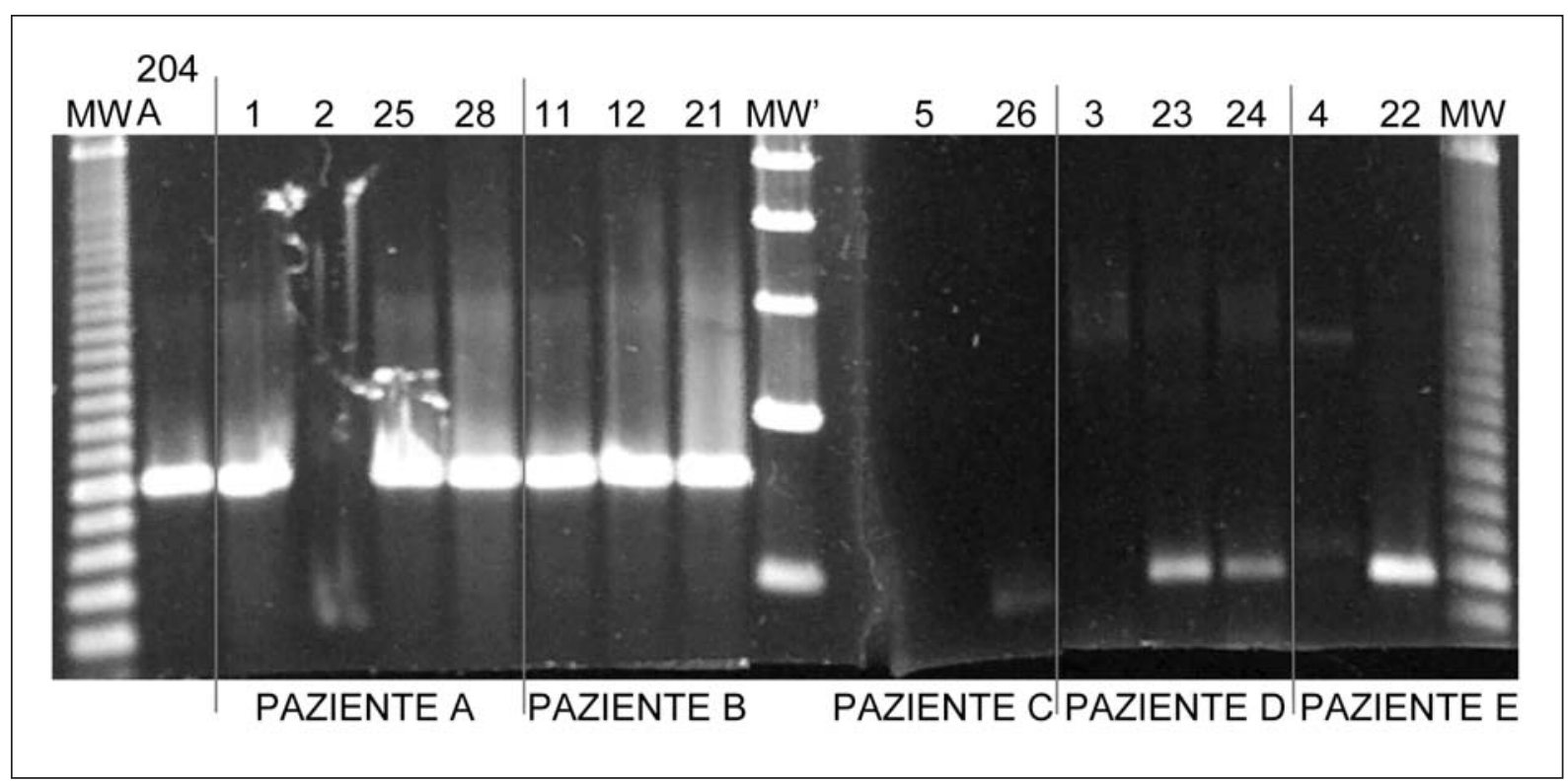

Figura II. Ricerca delle cassette geniche mediante amplificazione con primer 5'CSF e 3'CSL.

$8 \mu \mathrm{L}$ di amplificato sono stati separati in gel di agarosio 1\%; MW: 100 bp; MW': Kilobase.

La conferma della presenza del gene dell'integrasi è stata ottenuta tramite dot blot solo per i campioni 204A (controllo +), 1, 25, 28, 11, 12, 21.

Il sequenziamento dell'amplificato ha permesso di verificare che la cassetta presente è identica in tutti gli isolati.

Si noti come l'isolato BTS2, pur provenendo dallo stesso paziente di BTS1, non abbia l’integrone, e a distanza di anni si sia affermato il ceppo avente l'integrone stesso. 


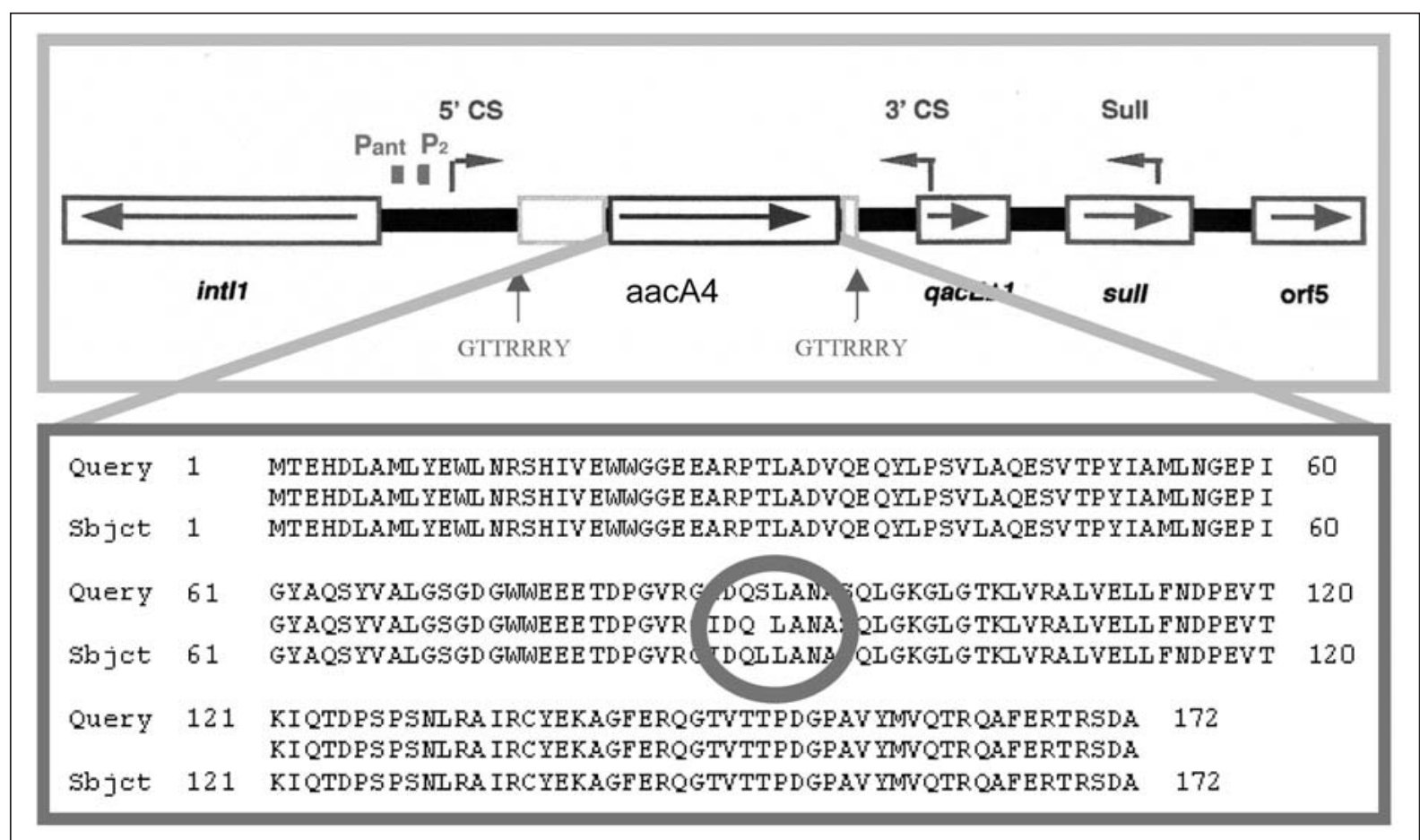

Figura III. Rappresentazione dellintegrone identificato in BTSI (a) e appaiamento della proteina Aac6'-Ib di BTSI-forma mutata (Query) con quella di A. baumannii 204A-wild tipe (Sbjct) (b).

La figura mostra come la mutazione puntiforme da -TTA a -TCA porta a un cambio amminoacidico (da Leucina a Serina) presente nella cassetta genica di tutti gli isolati di Bcc analizzati, rispetto alla cassetta wt di A. baumannii.

La mutazione già descritta da Shi e collaboratori (8) determina un livello di resistenza inferiore ad alcuni aminoglicosidi.

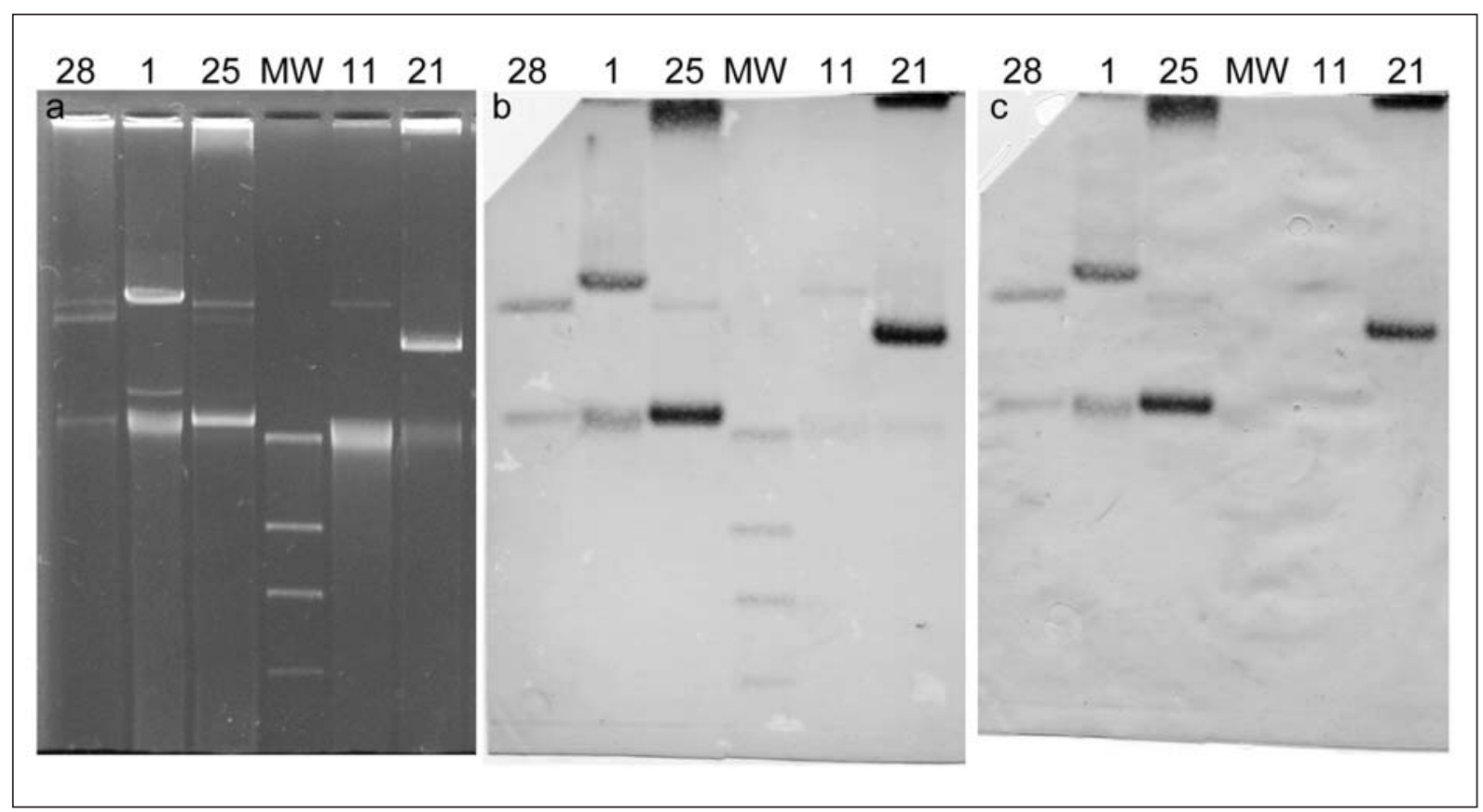

Figura IV. Elettroforesi e Southern blot con sonda omologa al gene Intl e sonda omologa al gene aacA4.

Separazione elettroforetica in gel di agarosio all'1\% dei plasmidi estratti dai ceppi Bcc risultati positivi alla presenza di integroni Southern blot con sonda omologa al gene Int1 marcata con digossigenina.

Southern blot con sonda omologa al gene aacA4 marcata con digossigenina dopo rimozione della sonda Int1 (1).

MW: DNA Molecular Weight Marker II marcato con digossigenina. 


\section{BIBLIOGRAFIA}

1. Boehringer Mannheim GmbH. The DIG system users manual for filter hybridization. Boehringer Mannheim GmbH, Biochemica, Mannheim, Germany 1993.

2. CLSI 2006 Performance Standards for antimicrobial susceptibility testing, 16th informational supplement, M100-S16.

3. Gombac F, Riccio ML, Rossolini GM, et al. Molecular characterization of integrons in epidemiologically unrelated clinical isolates of Acinetobacter baumannii from Italian hospitals reveals a limited diversity of gene cassette arrays. Antimicrobial Agents Chemotherapy 2002; 46(11): 3665-8.

4. Lévesque C, Piché L, Larose C, Roy PH. PCR mapping of integrons reveals several novel combinations of resistance genes. Antimicrobial Agents Chemotherapy. 1995; 39(1): 185-91.
5. Mahenthiralingam E, Campbell ME, Henry DA, Speert DP. Epidemiology of Burkholderia cepacia infection in patients with cystic fibrosis: analysis by randomly amplified polymorphic DNA fingerprinting. Journal Clinical Microbiology. 1996; 34(12): 2914-20.

6. Sambrook J, Fritsch E, Maniatis T. Molecular cloning; II edizione; 1989. COLD SPRING HARBOR LABORATORY PRESS.

7. Shaw KJ, Rather PN, Hare RS, Miller GH. Molecular genetics of aminoglycoside resistance genes and familial relationships of the aminoglycoside-modifying enzymes. Microbiology and Molecular Biology Reviews. 1993; 57: 138-63.

8. Shi WF, Jiang JP, Mi ZH. Relationship between antimicrobial resistance and aminoglycoside-modifying enzyme gene expressions in Acinetobacter baumannii. Chinese Medical Journal (Engl); 2005; 118(2): 141-5. 